\title{
Internal thoracic veins: Anatomy, plasticity and clinico-imaging relevance in small animal practice
}

\author{
Mario RICCIARDI ${ }^{1,2) *}$ and Alice CASALI ${ }^{3)}$ \\ ${ }^{1)}$ Ospedale Veterinario Gregorio VII. Piazza di Villa Carpegna 52, 00165-Roma, Italy \\ 2)Private practitioner, CT-MRI Support Service-70016, Noicattaro (BA), Italy \\ ${ }^{3)}$ Pingry Veterinary Hospital, Via Medaglie d'Oro 5-70126, Bari, Italy
}

\section{J. Vet. Med. Sci.}

82(9): 1358-1365, 2020

doi: 10.1292/jvms.20-0064

Received: 5 February 2020

Accepted: 15 July 2020

Advanced Epub:

24 July 2020

\begin{abstract}
The internal thoracic veins (ITVs) are small paired vessels located on the ventral surface of the thoracic cavity that drain the ventro-cranial abdominal wall, the ventro-lateral thoracic wall, the diaphragm and part of the mediastinum, conveying blood from these regions into the cranial vena cava. These vessels demonstrate a high level of anatomic plasticity and haemodynamic adaptability in both humans and small animals with blood flow impairment of the main abdominal and thoracic venous trunks. The ITVs may act as a natural bypass between the cranial and caudal venous system and between the portal vein and the cranial vena cava, depending on the level of the obstruction, giving rise to a wide spectrum of collateral pathways: intrathoracic cavo-caval, thoraco-abdominal cavo-caval, abdomino-thoracic cavo-caval, portocranial caval and lateral thoracic-azygos ITV collaterals. This paper provides a brief overview of the normal and pathologic anatomy of the ITVs described in dogs with cranial and caudal vena cava obstruction and portal hypertension as shown by CT angiography. Collateral ITV pathways need to be distinguished from other vascular anomalies in dogs, and their identification during routine CT studies could help radiologists to reach a more accurate diagnosis of caval or portal flow disturbance.
\end{abstract}

KEY WORDS: collateral circulation, computed tomography, dog, internal thoracic veins, vascular anatomy

In mammals, the venous blood flows in a unidirectional pathway towards the right atrium through two main vascular systems: the portal vein and the caval venous system.

The portal vein conveys blood from major abdominal organs (i.e., gastrointestinal tract, pancreas, spleen) to the liver for detoxification and then delivers it into the systemic circulation via hepatic veins and the caudal vena cava (CdVC) [3]. The caval system collects deoxygenated blood from all tissues of the body cranially and caudally to the heart, conveying it into the right atrium via the cranial vena cava (CrVC) and the CdVC [5]. The internal thoracic veins (ITVs) are small tributaries of the CrVC, located on the ventral surface of the thoracic cavity, which drain the ventro-cranial abdominal wall, the ventro-lateral thoracic wall, the diaphragm and part of the mediastinum.

Chronic obstructions or increases in blood flow resistance at any level of the portal and caval venous circulation (main trunks and their tributaries) lead to collateral vein formation to maintain venous drainage to the right atrium.

Although the CrVC, CdVC and portal vein may appear to work separately, there is a high plasticity between these three vascular systems and their branches, that is revealed in cases of blood flow disturbance. As many other vessels, the ITVs can be involved in several collateral circulation routes that can develop within four major pathways:

1) along a single large vein, bridging the patent segments of the obstructed vessel, as reported for the cavernous transformation of the portal vein [12] and for obstruction of the CdVC (cavo-caval shunt) [11];

2) through connection of different venous systems within a single region, as observed for abdominal acquired porto-caval shunts subsequent to portal hypertension [2] and for abdominal cavo-portal shunt in patients with CdVC obstruction [11];

3) through connection of the same venous system through different body regions, as observed in the deep and superficial venous collaterals bridging the caudal and cranial vena cava via the azygos vein in patients with CdVC obstruction [11];

4) through connection of different venous systems between different body regions, as observed in enlargement of the periesophageal venous plexus (esophageal varices) draining blood in the $\mathrm{CrVC}$ via the azygos vein in patients with portal 
hypertension [2].

Intrathoracic venous collaterals or connections between abdominal and thoracic regions by collateral circulation, which

demonstrate the higher level of plasticity of the vascular system, exploit two main venous routes: the azygos vein and the ITVs.

Despite several descriptions of collateral venous pathways involving the azygos vein having been published in the veterinary literature $[2,10]$, limited information is available on the possible involvement of the internal ITVs. Nowadays, the wide availability of computed tomography angiography (CTA) from multidetector-row CT (MDCT) scans in veterinary practice provide exhaustive depictions of large and small vessels throughout the whole body, allowing assessment of increasingly fine vascular anatomical details until now under-investigated.

This article offers an overview of the normal anatomy and acquired vascular anomalies of the ITVs in dogs with blood flow disturbance at different levels of the caval and portal venous systems, as determined by multidetector-row computed tomography angiography.

\section{NORMAL ANATOMY OF THE INTERNAL THORACIC VENOUS SYSTEM}

The internal thoracic venous system drains the ventro-cranial abdominal wall, the ventro-lateral thoracic wall, the diaphragm and part of the mediastinum, conveying the deoxygenated blood from these regions into the CrVC. From both sides, the ITVs originate from two main roots: 1) the cranial epigastric and cranial superficial epigastric veins and 2) the musculophrenic vein.

The cranial epigastric vein originates in the wall of the middle abdomen, at the level of the cranial abdominal mammae, and anastomoses with subcutaneous mammary branches of the cranial superficial epigastric veins. From this common origin the cranial epigastric vein passes dorsally to the rectus abdominis muscle and runs cranially towards the sternum. The cranial superficial epigastric veins run cranially in the same direction within the subcutaneous tissue (ventral to the rectus abdominis muscle) draining the mammary glands (cranial abdominal and thoracic mammae). At the level of the sternal xiphoid process, the cranial epigastric and cranial superficial epigastric veins join in a single vessel giving rise to one of the two main branches of the ITV.

The second main root of this vessel, the musculophrenic vein, originates from the ventro-lateral surface of the diaphragm and joins the ITV at the level of the latero-dorsal surface of the sternum (Fig. 1).

The ITVs run on both sides of the dorsolateral surface of the sternum, medially to the internal thoracic arteries and, along their course, receive the intercostal veins from the ventro-lateral inner surface of the thorax and small venous perforating branches (perforating veins) from the soft tissue of the pectoral region and thoracic mammary glands (Fig. 2). At the level of the second costal cartilage the ITVs run dorsally to enter the CrVC. In the cranial mediastinum, they receive the pericardiacophrenic veins, which are small venous vessels running from the cranial surface of the diaphragm along both side of the pericardial surface (adjacent to the course of the phrenic nerve) to enter the ITVs just before their caval insertion. In the cranial mediastinum the ITVs also collect small mediastinal veins and, in young animals, the thymic veins.

In dogs, the caval insertion of the ITVs can be unpaired or double. In cases of unpaired insertion, a single median trunk collects the two main vessels on both sides of the sternum end enters the ventral surface of the CrVC caudally to the brachiocephalic veins. In cases of double caval insertion, the left ITV enters the ventral surface of the left brachiocephalic vein independently of the right one, which enters the ventral surface of the CrVC [1, 5] (Fig. 2).

\section{PATHOLOGIC ANATOMY OF THE INTERNAL THORACIC VEINS}

\section{Intrathoracic cavo-caval collateral circulation}

In the case of chronic obstruction or increase in flow resistance at the level of the CrVC, the blood can reach the right atrium through a dorsal collateral pathway represented by enlargement of the periesophageal venous plexus draining into the azygos or portal vein (downhill varices) [2] or through a ventral collateral pathway using the ITVs. The ITVs collateral pathway provides a natural bypass from the cranial to the CdVC on the ventral and middle part of the thorax. Ventrally the ITVs enlarge and join the CdVC at the level of its caval foramen, running over the diaphragm surface (Fig. 3). In the middle part of the thorax multiple small tortuous mediastinal collaterals connect the $\mathrm{CrVC}$ from the brachiocephalic vein bifurcation to the CdVC forming a complex vascular network on each side of the heart surface. These vessels, which derive from the enlargement of the pericardiacophrenic veins, enter the CdVC at the level of caval foramen or via the phrenic vein [8] (Fig. 3).

In normal conditions close anatomical relationships are described between the venous drainage of the diaphragm and the internal thoracic and intercostal venous systems: the ventral phrenic veins anastomose on both sides with termination of the ITVs on the ventral diaphragmatic surface and with the intercostal veins on the lateral diaphragmatic surfaces. The ventral part of the intercostal veins from T8 to T13 also anastomose with the ITV and with their musculophrenic roots [4]. This diaphragmatic, peridiaphragmatic and intercostal venous net may enlarge in cases of CrVC obstruction mimicking an ITV-phrenic-caudal cava collateral pathway.

This large collateral pathway involving directly the ITV appears similar to the caval-mammary (internal thoracic)-phrenic pattern described in humans, in which blood flows from the internal thoracic vein to the inferior phrenic vein, thus entering the CdVC in close proximity to the diaphragm [6].

The small mediastinal pathway appears similar to that described in humans with superior vena cava syndrome, in which blood flows through the mediastinal, pericardial and pericardiacophrenic veins, draining towards the inferior phrenic veins and ending in the inferior vena cava $[6,8]$. 


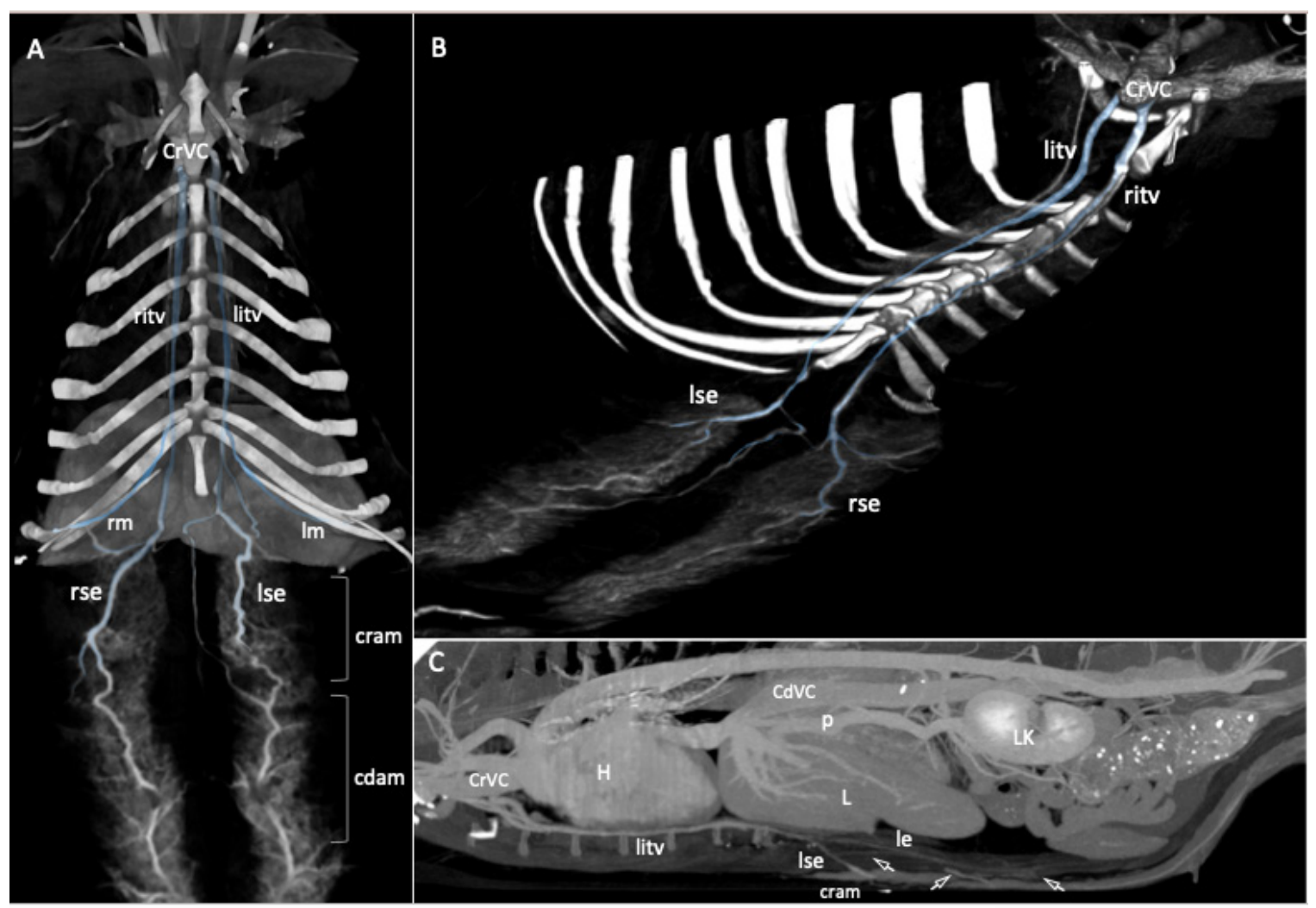

Fig. 1. Normal anatomy of the internal thoracic venous system of a 5-year-old female mixed-breed dog. A) Volume-rendered multidetector-row computed tomographic (MDCT) image of the thoracic region and abdominal mammae-ventral view. B) Volume-rendered MDCT image of the sternum and abdominal mammae, right caudodorsolateral view. C) Left parasagittal maximum intensity projection MDCT image of the thoracic and abdominal region after removal of the sternebrae. The internal thoracic veins (ITVs) (right, ritv; left, litv; blue in A and B) originate from two main roots: 1) the cranial epigastric (left, le in C) and cranial superficial epigastric veins (right, rse; left, lse in A and B) and 2) the musculophrenic veins (right, rm; left, $1 \mathrm{~m}$ in A). The ITVs run cranially on both sides of the dorsolateral surface of the sternum, medially to the internal thoracic arteries, and at the level of the second costal cartilage run dorsally to enter the cranial vena cava (A-C). CrVC, cranial vena cava; CdVC, caudal vena cava; p, portal vein; ritv, right ITV; litv, left ITV; rm, right musculophrenic vein; lm, left musculophrenic vein; rse; right cranial superficial epigastric vein; lse, left cranial superficial epigastric vein; le, left cranial epigastric vein; cram, cranial abdominal mammary gland; cdam, caudal abdominal mammary gland; empty arrows, subcutaneous branches of the cranial superficial epigastric veins; H, heart; L, liver; LK, left kidney.

In all these collateral pathways an inversion in the blood flow in the ITVs and pericardiacophrenic veins is expected.

\section{Thoraco-abdominal cavo-caval collateral circulation}

In the case of chronic obstruction or increase in flow resistance at the level of the CrVC, the blood from the upper body regions (forelimbs, neck, head) can reach the right atrium also through a long connection with the abdominal CdVC. This is exploited through the connection between the ITVs and the iliac veins via caudal superficial epigastric veins. In this condition a single large thoraco-abdominal venous pathway forms between the ITVs and the mammary veins (Fig. 4).

In humans this pattern of collateral circulation is seen in cases of superior vena cava stenosis with associated occlusion of the azygos vein and has been defined as a deep route of the abdominal wall collaterals. Other alternative superficial routes observed in humans are the lateral route, from the subclavian vein to the great (medial) saphenous vein (passing through the lateral thoracic, thoracoepigastric and superficial circumflex iliac vein) and a medial route, from the internal mammary vein to the great (medial) saphenous vein (passing through the superficial tributaries of internal mammary and para-umbilical veins and the superficial epigastric vein) [7].

In all these cases, an inversion of blood flow at the level of the ITVs is expected.

\section{Abdomino-thoracic cavo-caval collateral circulation}

In the case of obstruction of the CdVC, a superficial collateral pathway has been described to carry venous blood from the pelvic limbs and caudal abdomen to the right atrium. Multiple subcutaneous tortuous vessels drain blood from the deep circumflex iliac veins, anastomosing with the caudal epigastric and caudal superficial epigastric veins ending in the ITVs. Alternatively, the circumflex iliac veins can join the 11th intercostal veins to end at the azygos vein [11]. This collateral pathway is similar to the thoraco-abdominal cavo-caval collateral circulation described for obstructions at the level of the CrVC but with inverted blood flow. 

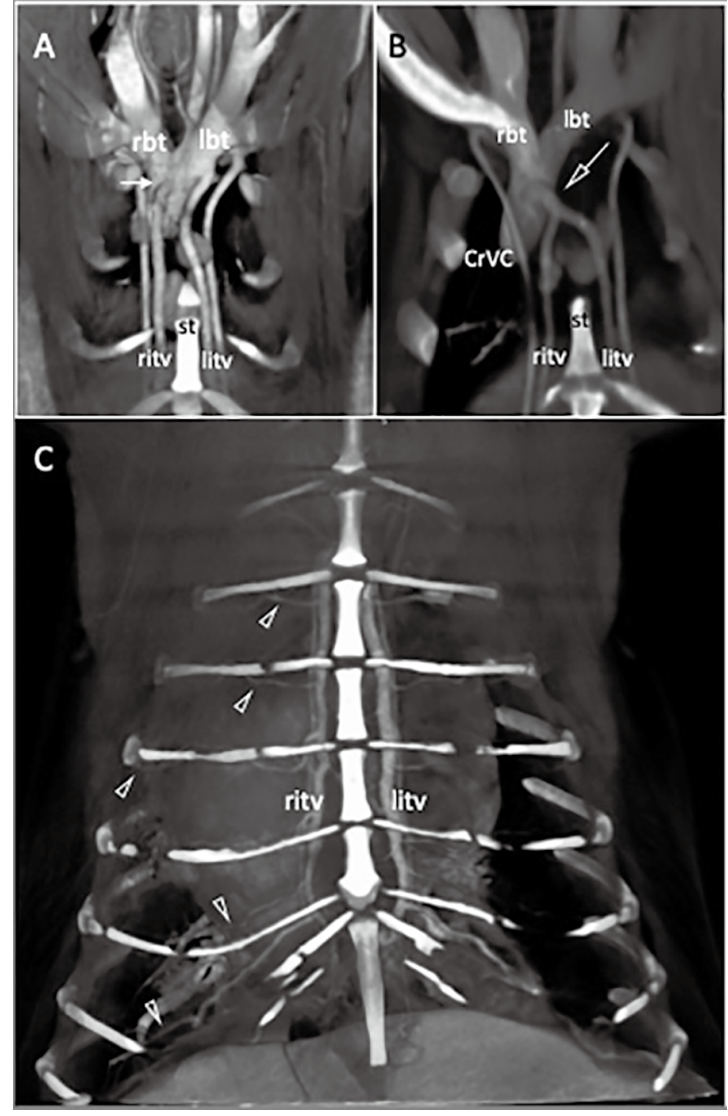

Fig. 2. Normal anatomy of the internal thoracic venous system of a 5-year-old female mixed-breed dog. A, B) ventral volume-rendered multidetector-row computed tomographic (MDCT) images of the cranial vena cava ( $\mathrm{CrVC}$ ) showing in detail the variations of the caval insertion of the internal thoracic veins (ITVs): A) double caval insertion. B) Unpaired insertion with a single median trunk (empty arrow). C) Ventral volume-rendered MDCT image of the thoracic region showing a detail of the insertion of the intercostal veins (arrowheads) from the ventro-lateral inner surface of the thorax into the ITVs. Ventrally, the ITVs receive small perforating veins from the soft tissue of the pectoral region and thoracic mammary glands (not detectable under normal conditions because of the small dimensions of these vessel compared to the spatial resolution of the CT scan). St, Sternum; litv, left ITV; ritv, right ITV; lbt, left brachiocephalic vein; rbt, right brachiocephalic vein; arrow, entrance of the right ITV into the ventral surface of the CrVC.

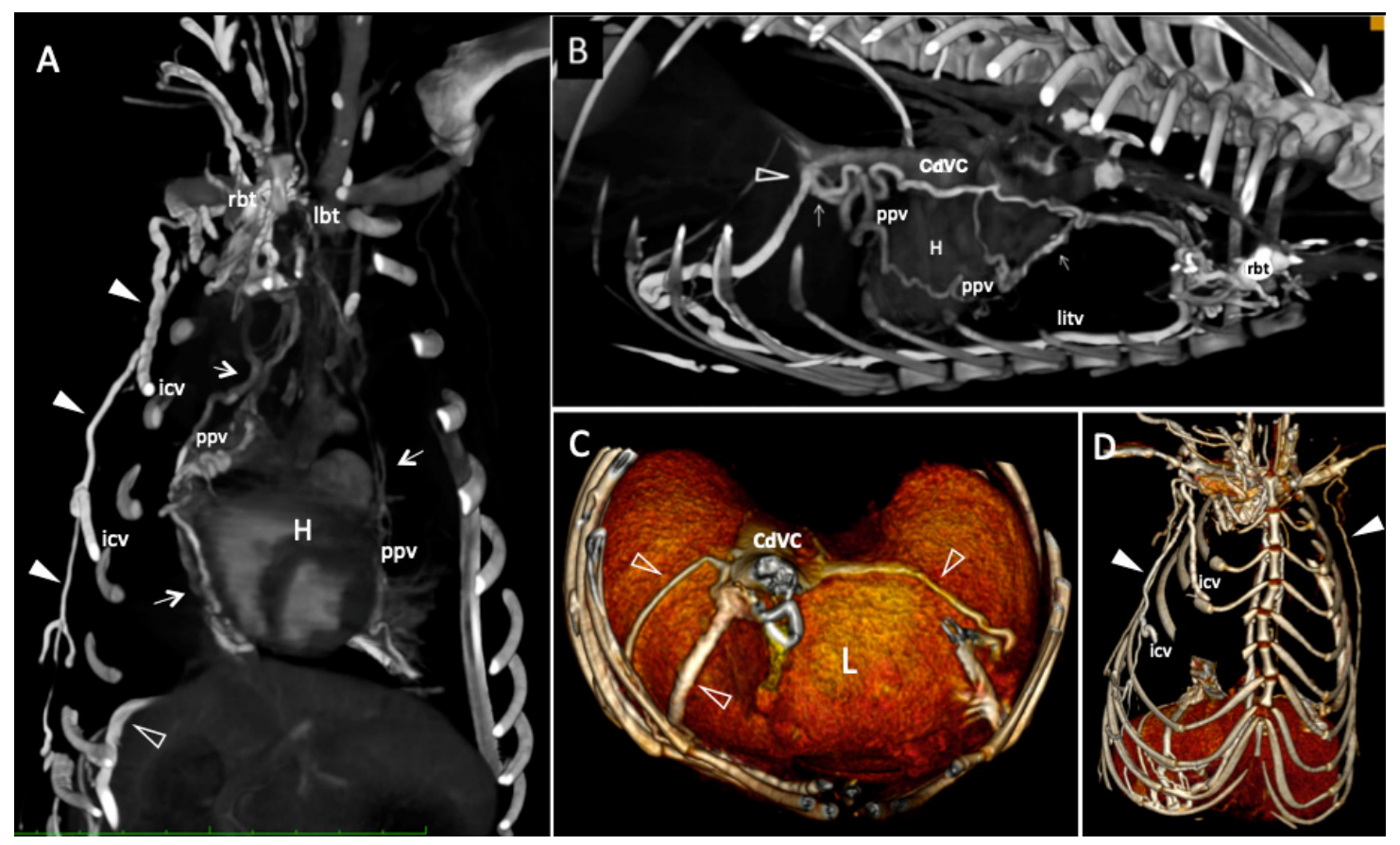

Fig. 3. An 8-year-old neutered female Yorkshire terrier with mediastinal neoplasm and subsequent total cranial vena cava (CrVC) invasion and obstruction. Detail of peridiaphragmatic, intercostal, mediastinal and internal thoracic collaterals (intrathoracic cavo-caval collateral circulation). A) Ventral and B) Right parasagittal thick-slab multiplanar reformatted contrast-enhanced CT image of the thorax. C) Volume-rendered multidetectorrow computed tomographic (MDCT) image of the thoracic surface of the diaphragm. D) Ventral volume-rendered MDCT image of the thorax. Multiple small tortuous vessels (arrows), resembling varices, connect the right (rbt) and left (lbt) brachiocephalic venous trunks with the caudal vena cava (CdVC) at the level of the caval foramen. These vessels, forming a complex vascular network on each side of the heart surface, derive from the enlargement of the pericardiacophrenic veins (ppv, A and B). The internal thoracic veins (ITVs) appear enlarged and connected to the CdVC through multiple large venous vessels running over the diaphragm from both sides, resembling the enlarged ventral phrenic veins and musculophrenic veins (empty arrowheads). Enlargement of the lateral thoracic veins (LTVs-filled arrowheads A and D), joining the intercostal veins (icv, A and D), is also seen. $\mathrm{H}$, heart; L, liver. 


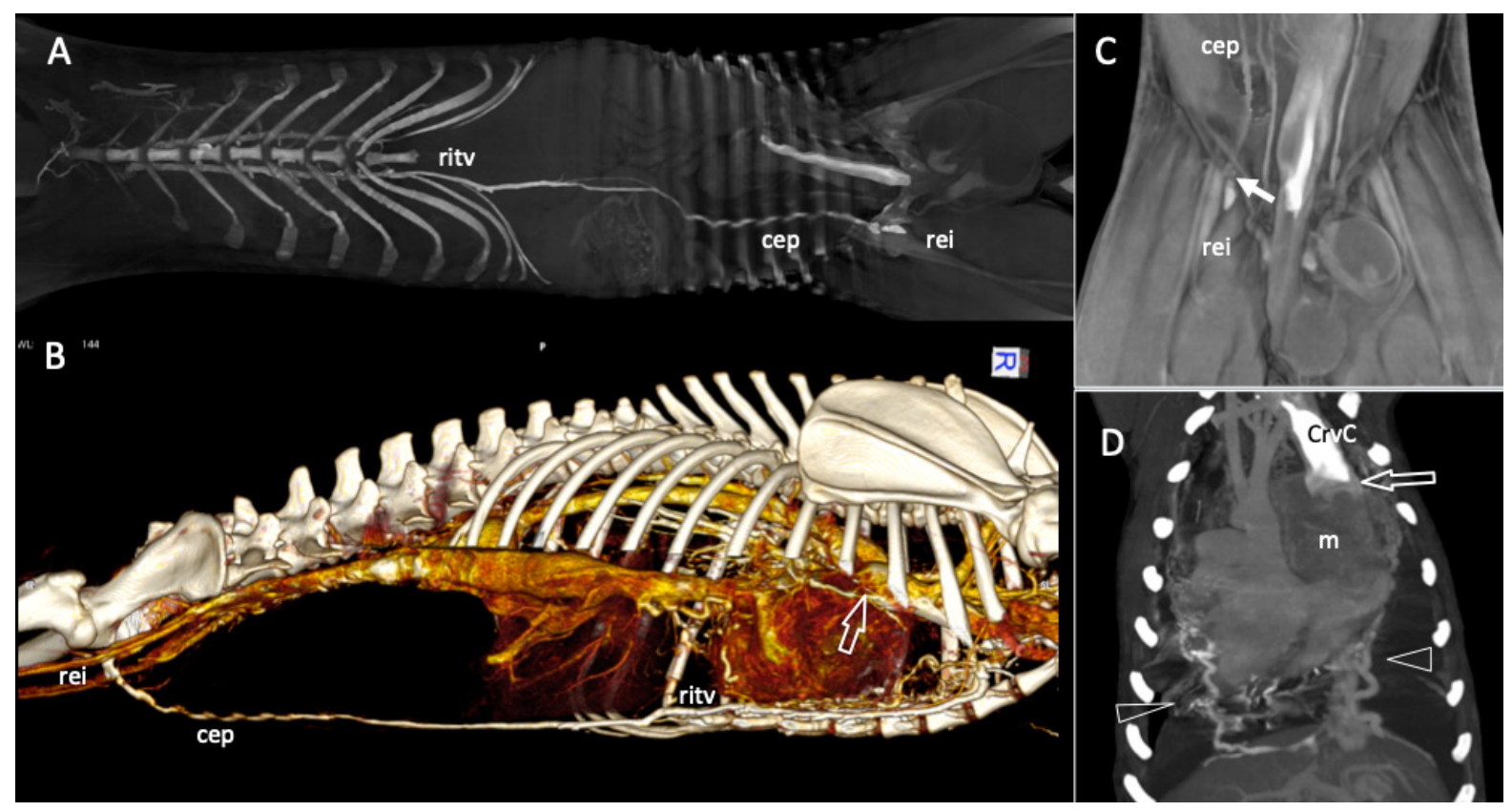

Fig. 4. A 9-year-old male English Setter dog with myocardial neoplasm (m, D) and subsequent cranial vena cava (CrVC) invasion and obstruction at the level of its insertion into the right atrium (empty arrows, B and D). Detail of the thoraco-abdominal cavo-caval collateral circulation. A) Ventral thick-slab multiplanar reformatted contrast-enhanced CT image of the thorax and abdomen (horizontal stripes on the abdomen are due to breathing artefacts). B) Right lateral volume-rendered CT image of the thorax and abdomen. C) Ventral volume-rendered CT image of the inguinal region. D) Dorsal maximum intensity projection CT image of the thorax. In the thoraco-abdominal cavo-caval collateral pathway the right internal thoracic vein (ritv) joins the right external iliac vein (rei) via the caudal superficial epigastric vein (cep). Image $\mathrm{C}$ shows in detail the origin of the caudal superficial epigastric vein (cep) from the right external iliac vein (rei) (arrow). This patient showed concomitant mediastinal collaterals (arrowheads).

\section{Porto-cranial caval collateral circulation}

Acquired collateral circulation of the portal vein is a well-known compensatory mechanism that develops in the case of obstruction or increased resistance to the portal flow towards the liver to alleviate portal hypertension (PH). Commonly acquired portal collaterals develop between the main portal branches and the main tributaries of the CdVC as large collaterals or small varices [2]. The connection between the portal vein and the cranial caval system in PH patients develops through the azygos vein (spleno-azygos acquired portosystemic-shunt-APPS) and through the ITVs.

The spleno-azygos APSS has been described both in humans and small animals but its real origin and differentiation from a simple enlargement of the left gastric and oesophageal veins (cardiac branches of the left gastric vein and oesophageal branches of the azygos vein) is still debated $[3,10]$.

The connection between portal vein and CrVC via ITVs has been recently described in a dog with PH subsequent to a primary hypoplasia of the portal vein. In this patient a tortuous vessel originated from the portal vein before its intra-hepatic division, ran ventrally through the abdominal wall and joined the left ITV to reach the CrVC [9] (Fig. 5).

Interestingly, this porto-cranial caval collateral circulation has been reported in humans with PH. In these cases, the portal system joins the internal mammary veins via the para-umbilical veins of the abdominal wall and drain into the superior vena cava. In another abdominal wall collateral route reported in humans, from the portal vein the para-umbilical veins drain (via the inferior and superficial epigastric veins) into the external iliac and great (medial) saphenous veins [7].

\section{Obstruction of the ITVs: lateral thoracic-azygos collaterals}

In the case of obstruction of the CrVC and caval termination of the ITVs, as may be observed in patients with large expansive masses in the cranial mediastinum, venous drainage from the upper body regions to the right atrium is ensured by a collateral circulation exploiting a minor venous net of the thoracic walls: the lateral thoracic veins (LTVs). LTVs in normal conditions drain the latero-dorsal part of the thoracic walls and the thoracic mammae (Fig. 6). When the ITVs are compressed and cannot participate in collateral routes, venous blood from the neck and forelimbs runs from the brachiocephalic trunks into the enlarged LTVs on both side of the thoracic wall lateral to the ribs. Along the ribs, the LTVs anastomose with the intercostal veins and run dorsally on the inner surface of the thoracic wall to end in the azygos vein. (Figs. 7 and 8). In the subclavian-lateral thoracic-azygos pathway an inversion of the venous blood flow can be hypothesized. Enlargement of a thin subcutaneous venous net (superficial tributaries of the ITVs) may be observed in the pectoral region to contribute in this collateral pattern. In humans, LTV collateral routes are described as possible acquired collateral pathways in patients with superior vena cava and portal obstruction [7]. 


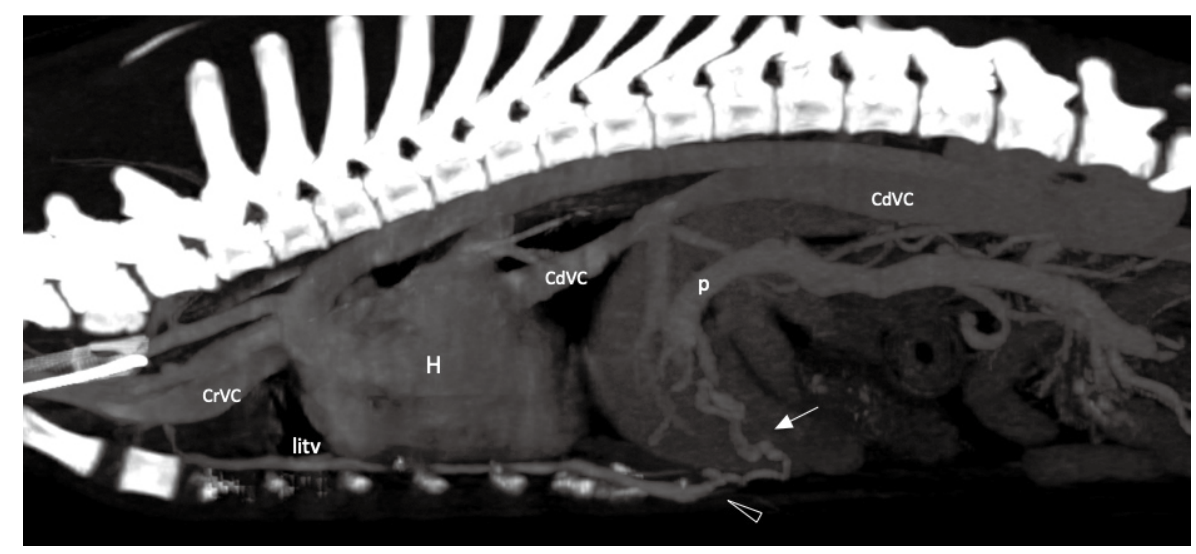

Fig. 5. A 6-months-old female Rottweiler with portal hypertension ( $\mathrm{PH})$ and acquired porto-cranial caval collateral circulation from portal vein (p) to the cranial vena cava (CrVC) via the left internal thoracic vein (ITV). Left parasagittal Maximum Intensity Projection (MIP) CT angiography of cranial abdomen and thorax. A tortuous vessel (arrow) originates from the portal vein (p) before its intra-hepatic branches, courses ventrally and joins the left ITV (litv) to reach the CrVC. Arrowhead indicates the point of connection between the portal vein (p) and left ITV (litv). CdVC, Caudal vena cava; H, Heart.
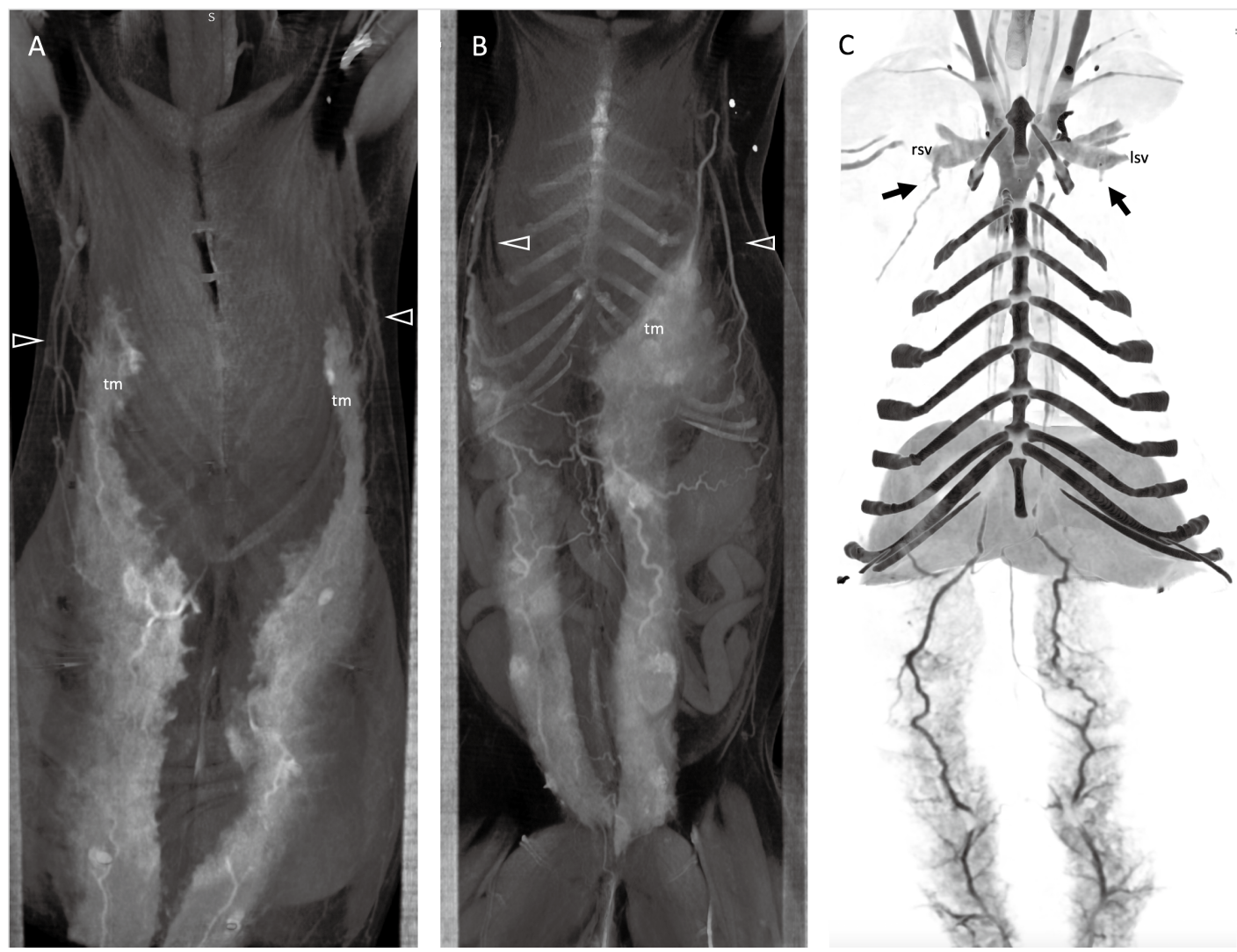

Fig. 6. Normal anatomy of the lateral thoracic veins (LTVs). A, B) Ventral volume-rendered multidetector-row computed tomographic (MDCT) images of different medium-sized female mixed-breed dogs showing detail of the LTVs (arrowheads) draining the thoracic mammary glands (tm). C) Ventral inverse maximum intensity projection (MIP) MDCT image of the thoracic region and abdominal mammae in a 5-year-old female mixed-breed dog. Black arrows point to the insertion of the LTVs into the left (lsv) and right (rsv) subclavian veins.

\section{CONCLUSIONS}

In recent years, with the diffusion of modern MDCT scanners into veterinary practices, there is an increasing availability of high-quality near-isotropic CT angiographic studies that allow a more detailed evaluation of the vascular system in both normal 

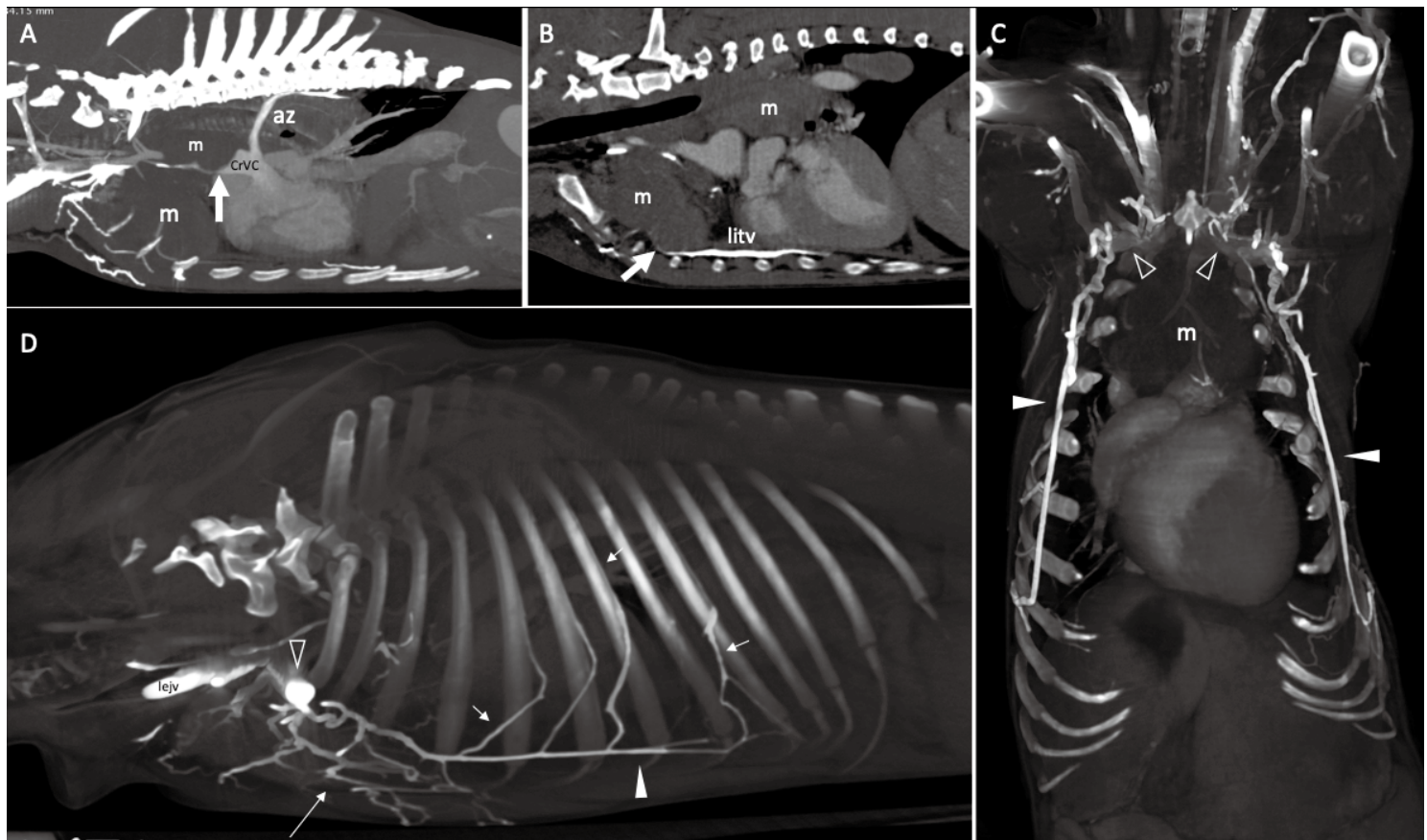

Fig. 7. Enlargement of the lateral thoracic veins (LTVs) in a 6-year-old male Rottweiler dog with cranial vena cava (CrVC) and internal thoracic veins (ITVs) compression subsequent to a large mediastinal neoplasm. (A) Right parasagittal MIP and (B) left parasagittal multiplanar reformatted multidetector-row computed tomographic (MDCT) images of the thorax showing the large mediastinal mass (m) compressing the CrVC and the left ITV (litv) (right ITV not shown). The arrows in images A and B indicate the point of compression of the CrVC and the left ITV (litv). The azygos vein (az) is not compressed. (C) Ventral volume-rendered MDCT image of the thorax showing the enlarged LTVs (filled arrowheads) running along the thoracic walls from the subclavian veins (empty arrowheads). (D) Left lateral volume-rendered MDCT image of the thorax showing the enlarged left lateral thoracic vein (filled arrowhead) connecting the left subclavian vein (empty arrowhead) with the intercostal veins (short arrows). Enlargement of the subcutaneous venous net in the pectoral region is also visible (long arrow). Lejv, left external jugular vein.

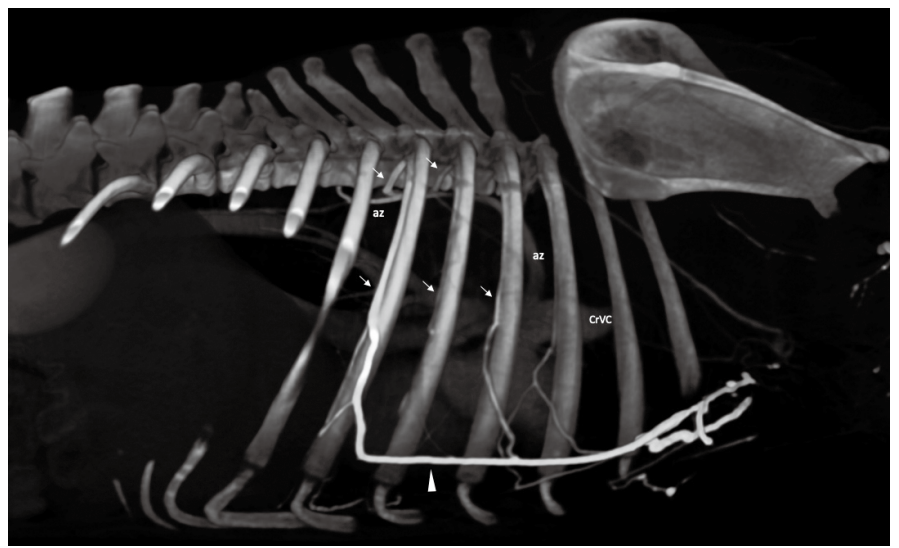

Fig. 8. Right lateral volume-rendered multidetector-row computed tomographic (MDCT) image of the thorax of the same dog as in Fig. 7 showing detail of the connection between the lateral thoracic and azygos veins via the intercostal veins to convey venous blood from the neck to the right atrium. The filled arrowhead points to the right lateral thoracic vein; the arrows point to the intercostal veins and their connection to the azygos vein (az); CrVC, cranial vena cava. and pathologic conditions. In this scenario, the ITVs have received increased attention in recent veterinary literature because of their involvement in different collateral vascular routes in patients with impairment of the portal or caval blood flow. The ITVs may act as a natural bypass between the cranial and caudal venous system and between the portal vein and the CrVC depending on the level of venous blood flow disturbance. The wide spectrum of possible collateral pathways of the ITVs, schematically summarized in Fig. 9, testifies the high level of plasticity and adaptability of the venous system of dogs from both an anatomical and a haemodynamic point of view. Finally, from a clinicodiagnostic point of view, the identification of collateral pathways of the ITVs, as an important ancillary finding during routine CT studies, could help radiologists reach a more accurate diagnosis of caval or portal flow disturbance.

ACKNOWLEDGMENTS. The first author wishes to thank the staff of the Pingry Veterinary Hospital, Bari, Italy for 10 years of mutual scientific enrichment and the staff of Gregorio VII Veterinary Hospital, Rome, Italy for assistance with data collection. 


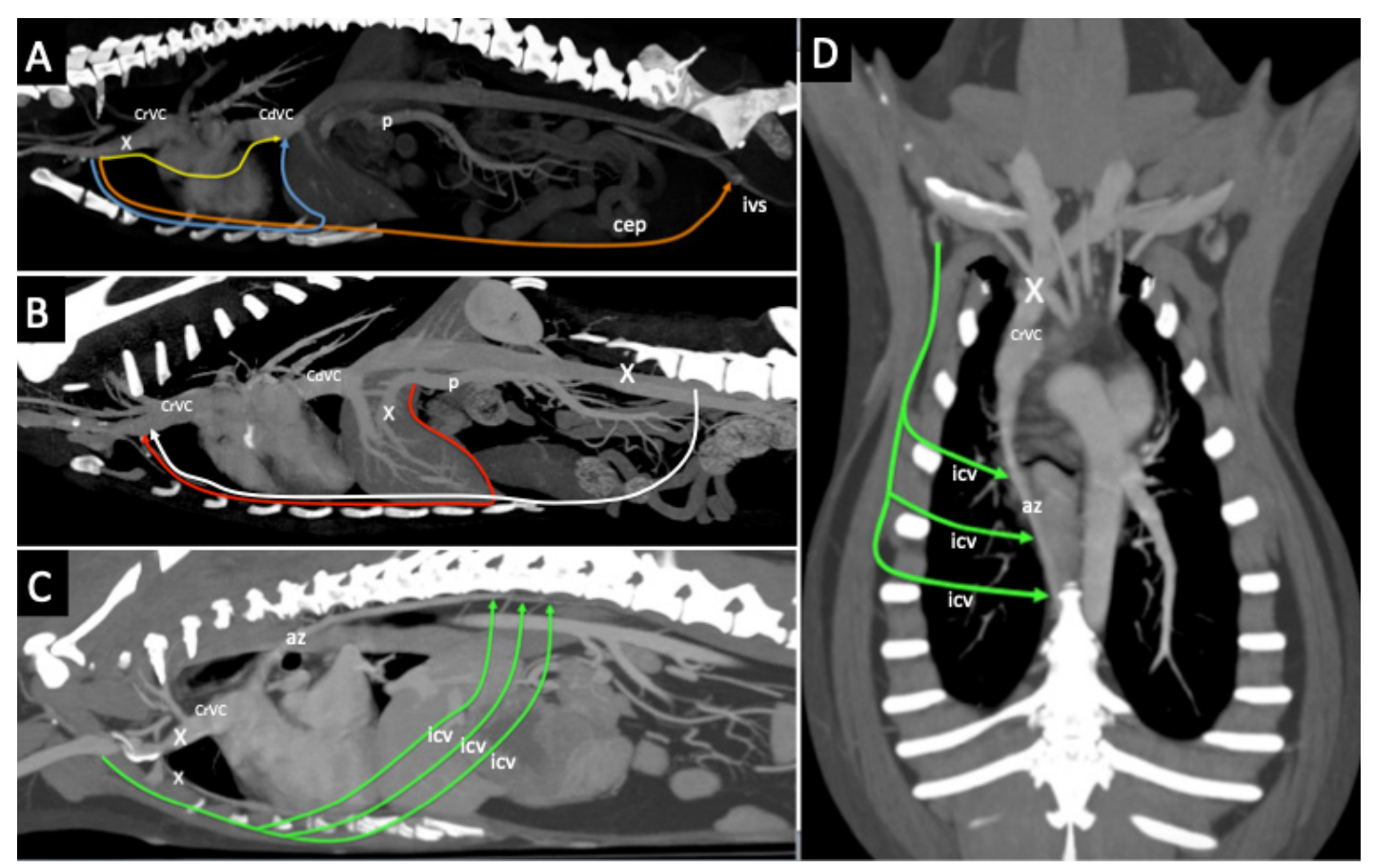

Fig. 9. Schematic representation of the different collateral pathways involving the internal thoracic veins (ITVs) in dogs with obstruction of the cranial vena cava $(\mathrm{CrVC})$. (A) In the intrathoracic cavo-caval collateral circulation the ITVs connect the CrVC with the CdVC at the level of its caval foramen (blue arrow). A small vascular network can join the same caval points through the mediastinal, pericardial, and pericardiacophrenic veins running on each side of the heart (yellow arrow). In the thoraco-abdominal cavo-caval collateral circulation the ITVs connect the cranial and caudal caval systems via caudal superficial epigastric veins (cep) and the iliac veins (ivs) (orange arrow). (B) In the abdomino-thoracic cavo-caval collateral circulation the blood flows from the caudal vena cava (CdVC) to the CrVC through connection of the deep circumflex iliac veins with the caudal epigastric and caudal superficial epigastric veins ending in the ITVs (white arrow). In the portocranial caval collateral circulation the portal vein (p) joins the ITVs to allow portal blood to reach the CrVC (red arrow). (C, D) In the lateral thoracic-azygos collateral pathway, when the ITVs and CrVC are compressed or obstructed, the LTVs convey blood from the brachiocephalic trunks in the azygos vein (az) via the intercostal veins (icv) (green arrows). For each scheme the arrowheads indicate blood flow direction. The white X's indicate the points of vascular obstruction/compression.

\section{REFERENCES}

1. Barone, R. 2003. Anatomia comparata dei mammiferi domestici. Vol. 5: Angiologia. Parte seconda: vene e sistema linfatico, 3rd ed. Edagricole, Bologna.

2. Bertolini, G. 2010. Acquired portal collateral circulation in the dog and cat. Vet. Radiol. Ultrasound 51: 25-33. [Medline] [CrossRef]

3. Bertolini, G. 2019. Anomalies of the portal venous system in dogs and cats as seen on multidetector-row computed tomography: an overview and systematization proposal. Vet. Sci. 6: 1-17. [Medline]

4. Comtois, A., Gorczyca, W. and Grassino, A. 1987. Anatomy of diaphragmatic circulation. J. Appl. Physiol. 62: 238-244. [Medline] [CrossRef]

5. Evans, H. E. and DeLahunta, A. 2013. Miller's Anatomy of the Dog, 4th ed. Saunders Elsevier, St. Louis.

6. Kapur, S., Paik, E., Rezaei, A. and Vu, D. N. 2010. Where there is blood, there is a way: unusual collateral vessels in superior and inferior vena cava obstruction. Radiographics 30: 67-78. [Medline] [CrossRef]

7. Miranda Orella, L., Mellado, J.M., Yanguas Barea, N., Solanas Alava S., Larrosa López, R. and Martín Cuartero, J. 2010. Collateral pathways of the abdominal wall: anatomical review and pathologic findings at 64-slice multidetector CT angiography. doi.org/ 10.1594/ecr2010/C-3062.

8. Ricciardi, M. and Lanci, M. 2017. Acquired collateral venous pathways in a dog with cranial vena cava obstruction. J. Vet. Med. Sci. 79: 17721775. [Medline] [CrossRef]

9. Ricciardi, M. 2016. Splenophrenic portosystemic shunt in dogs with and without portal hypertension: can acquired and congenital porto-caval connections coexist? Open Vet. J. 6: 185-193. [Medline] [CrossRef]

10. Ricciardi, M. 2017. Unusual haemodynamics in two dogs and two cats with portosystemic shunt - implications for distinguishing between congenital and acquired conditions. Open Vet. J. 7: 86-94. [Medline] [CrossRef]

11. Specchi, S., d'Anjou, M. A., Carmel, E. N. and Bertolini, G. 2014. Computed tomographic characteristics of collateral venous pathways in dogs with caudal vena cava obstruction. Vet. Radiol. Ultrasound 55: 531-538. [Medline] [CrossRef]

12. Specchi, S., Pey, P., Ledda, G., Lustgarten, M., Thrall, D. and Bertolini, G. 2015. Computed tomographic and ultrasonographic characteristics of cavernous transformation of the obstructed portal vein in small animals. Vet. Radiol. Ultrasound 56: 511-519. [Medline] [CrossRef] 